\title{
Cooperation between Local and Global Approaches to Register Brain Images
}

\author{
Pierre Hellier and Christian Barillot \\ IRISA, INRIA-CNRS, Campus de Beaulieu, 35042 Rennes cedex, France \\ phellier@irisa.fr, cbarillo@irisa.fr
}

\begin{abstract}
In this paper, we investigate the introduction of cortical constraints for non rigid inter-subject brain registration. We extract sulcal patterns with the active ribbon method, presented in 10. An energy based registration method [7] makes it possible to incorporate the matching of cortical sulci, and express in a unified framework the local sparse similarity and the global "iconic" similarity. We show the objective benefits of cortical constraints on a database of 18 subjects, with global and local measures of the quality of the registration.
\end{abstract}

\section{Introduction}

During the last few years, the development of electronic brain atlases has emerged by overcoming some limitations of traditional paper-based atlases. In particular, nonlinear registration methods were developed. They concentrate on the modeling of the interindividual morphological variability by the design of deformation models able to give an account of this variability [14. The development of intersubject registration methods is motivated by the possibility to match segmentation and labeling of anatomical structures from one template to one particular subject. An assumption is made that if a template (atlas) exists, the template labels can be deformed into another subject, under the assumption that there is a total relation between the points of the atlas (source) and the points of the studied subject (destination). This objective has been pursued for a long time in medicine and was traditionally treated by paper atlases with generally rather simple transformations. The most known example is the atlas of Talairach with its famous AC-PC referential and its related proportional squaring [21].

Another objective is to use this same framework for a better interpretation of cerebral functions observed through brain functional imaging (SPECT, PET, MEG/EEG, fMRI). Indeed, the links between anatomy and functional organization are often badly known: the superimposition of multi-individual neurofunctional recordings on the same anatomy may be of great interest to better understand the human brain functional organization. In this case, inherent anatomical variability between individuals may disturb this interpretation. Therefore, spatial normalization may be used to address directly the functional 
variability (under the assumption that two variabilities are not correlated). A better knowledge of this anatomy-function relationship is of great interest for the researcher in cognitive neuroscience, as well as for the surgeon and the neurologist who intend to delineate relevant functional areas before intervention in that region.

In this paper, we propose a unified framework for non rigid registration of brains, combining a global registration approach with sparse constraints. We explicitely use it with cortical sulci constraints in that case.

\section{Related Work}

An increasing number of authors study this registration problem. As it would be a gargantuan task to quote them all, we refer the reader to [12] for a complete survey on that subject. These methods are generally divided into two groups: "iconic" methods, that rely generally on the matching of voxels having the same luminance, and "geometric" methods that rely on the extraction and matching of landmarks. Very few methods do really make cooperation between these two classes of matching features. Gee et al. [6] have proposed a Bayesian unified framework to this problem but without experimentations on real 3D data. The problem is in fact to find a transformation which more or less conserves the topology of the brain while being able to adapt the transformation to local topological changes. For instance, a cortical sulcus may be present in one segment for one subject while being split in three parts for a second one, or even absent for a third subject. These variations can even be observed between two hemispheres of a single subject .

Many applications where brain warping is needed are facing this problem. There are some strong arguments to control non-rigid matching of brain data by local cortical landmarks. Recently Collins et al. 43. investigated the introduction of sulcal constraints, which have been introduced on the basis of a chamfer distance between corresponding sulci of the source and target volumes. The evaluation on real data have shown first the important lack of precision in the cortex of methods dealing with image intensities only, and also the benefits that can be expected with local cortical constraints. However, in that case sulcal constraints have been introduced without any specific formal framework, only the orthogonal projection of a sulcus onto its correspondent is used, as shown in the figure 1

Other works use also cortical constraints for non-rigid registration [23,24, where the surface deformation takes only into account the sulcal constraints detected on the brain external surface. Chui et al. 2 incorporates in the registration process scattered constraints such as sulcal points manually extracted by an expert. 


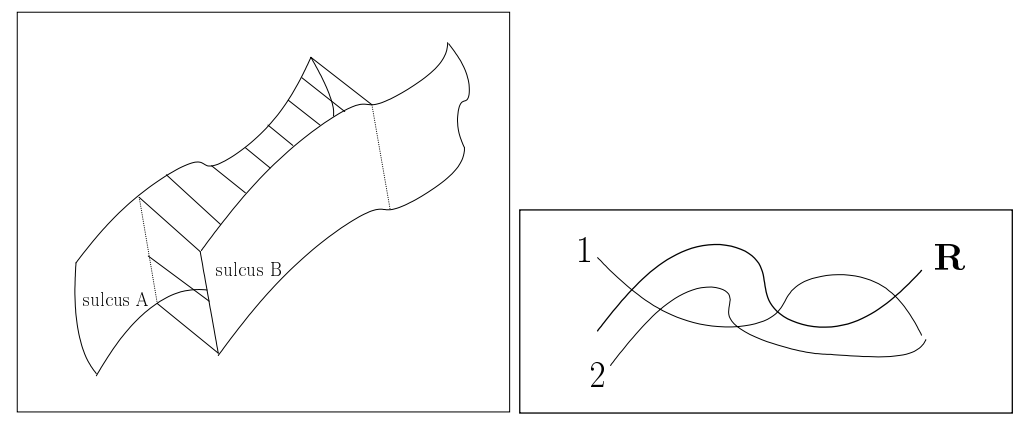

Fig. 1. Left: Integration of sulcal constraints using a chamfer distance criterion. Only orthogonal projections are retained for the registration constraints. An ICP matching algorithm would give similar results. Right: The sulcus $\mathrm{R}$ is the reference sulcus, and sulci 1 and 2 are deformed sulci that should ideally match the reference sulcus. Which one is the best?

\section{Energy-Based Registration Framework}

Formulation: In that section, we describe very briefly our previous work, on which the registration method is based. More details can be found in 7].

The registration process is expressed as the minimization of a cost function depending on two terms: a similarity measure, chosen as the optical flow [8], and a regularization term. Therefore the cost function to be minimized can be expressed as:

$$
U(\boldsymbol{w} ; f)=\sum_{s \in S}\left[\boldsymbol{\nabla} f(s, t) \cdot \boldsymbol{w}_{s}+f_{t}(s, t)\right]^{2}+\alpha \sum_{<s, r>\in \mathcal{C}}\left\|\boldsymbol{w}_{s}-\boldsymbol{w}_{r}\right\|^{2},
$$

where $s$ is a voxel of the volume, $t$ is the index of the volumes, $f$ is the luminance function, $\boldsymbol{w}$ is the expected $3 D$ displacement field, $S$ is the voxel lattice, $\mathcal{C}$ is the set of neighboring pairs and $\alpha$ controls the balance between the two energy terms.

To overcome some classical difficulties of that formulation (large displacements, noise and intensity inhomogeneities, discontinuities), we introduce two robust estimators, one for the data term $\left(\rho_{1}\right)$ and the second one for the regularization term $\left(\rho_{2}\right)$. The minimization is therefore equivalent to the minimization of the augmented function, noted $\stackrel{\star}{U}$ :

$$
\begin{aligned}
\stackrel{\star}{U}(\boldsymbol{w}, \delta, \beta ; f) & =\sum_{s \in S} \delta_{s}\left(\nabla f(s, t) \cdot \boldsymbol{w}_{s}+f_{t}(s, t)\right)^{2}+\psi_{1}\left(\delta_{s}\right) \\
& +\alpha \sum_{<s, r>\in \mathcal{C}} \beta_{s r}\left(\left\|\boldsymbol{w}_{s}-\boldsymbol{w}_{r}\right\|\right)^{2}+\psi_{2}\left(\beta_{s r}\right),
\end{aligned}
$$


where $\delta_{s}$ and $\beta_{s r}$ are auxiliary variables acting as weights. This cost function has the advantage to be quadratic with respect to $\boldsymbol{w}$. Furthermore, when a voxel $s$ does not validate the model, the corresponding weight $\delta_{s}=\phi_{1}^{\prime}\left(\left[\boldsymbol{\nabla} f(s, t) \cdot \boldsymbol{w}_{s}+\right.\right.$ $\left.f_{t}\right]^{2}$ ) decreases, making this formulation more robust. More details may be found in 7 .

Multiresolution and multigrid minimization: In order to cope with large displacements, a classical incremental multiresolution procedure is used (successive Gaussian smoothing and subsampling). At each resolution level, a multigrid minimization based on successive partitions of the initial volume is achieved. At each grid level, a 12-dimension parametric increment field is estimated over each cube of the grid. The resulting field is a rough estimate of the desired solution, and it is used to initialize the next grid level. This hierarchical minimization strategy improves the quality and the convergence rate.

\section{Extraction of Local Cortical Constraints}

\subsection{Related Work: Sulci Extraction}

Descriptive anatomy of the cerebral cortex is based on its subdivision in a set of sulci and gyri. MRI accurately represents this in-vivo cortical anatomy, and is used in the daily practice in morphological and functional brain imaging. In the context of this paper, these sulci serve as cortical landmarks to constrain the registration process. The sulci segmentation and identification task is a real three-dimensional data analysis issue. There are two main issues within this recognition topic : one addressing the problem of feature extraction for the segmentation of sulci, and one addressing the problem of the identification of cortical structures (lobes, gyri, sulci). Some methods dealing with the problem of representing cortical sulci define a sulcus from its structural information, basically a cortical fold filled by CSF 131819202425 . Other methods define a sulcus from its shape; basically a sulcus is a highly convoluted shape 1022. For the recognition task, the identification of cortical regions like lobes, gyri or sulci can use the atlas matching paradigm [11119] or be based on a symbolic/numeric data fusion scheme using probabilities [1117] or heuristics [18. One problem of these methods is the great variability of the cortical folds and the difficulty in analyzing them hierarchically (even if statistical maps can be used for a gross automatic labeling of some major sulci [11] or even neural networks based on a learning data set [17]).

\subsection{Modeling of Sulci Using Active Ribbons}

A compact numerical description of a sulcus can be obtained by modeling this sulcus with a surface representing its deep part. The method we use is based on the active contour paradigm evolving from a $1 \mathrm{D}$ curve located at the external part of the brain to a $2 \mathrm{D}$ surface modeling the median axis of the sulcus. In order 
to characterize sulci from gyri, the curvature of iso-intensity surfaces belonging to the cortical regions is computed. The operator used to compute curvature information is a $3 \mathrm{D}$ extension of the Lvv operator. Then, a compact and a parametric description of a sulcus is obtained by a median surface representing the buried part of this sulcus.

\subsection{Matching of Sulci}

The cortical sulci are extracted using the algorithm described in [10].

Contiguous Sulci: The sulci are modeled with B-splines, which facilitates their numerical manipulation. In particular, the resampling of these shapes is possible on each axis. For the registration of a subject $A$ towards a subject $B$, we consider the homologous sulci between these two subjects. For each pair of homologous sulci, the sulcus containing with fewer control points is resampled so that the two homologous sulci are finally described by the same number of points. Then, we explicitly put in correspondence the control points of these two sulci. This is summarized on the figure 2 On the support of the constraint sulci of the source volume, we thus define a sparse constraint deformation field which can be integrated thereafter in the estimate.

Interrupted Sulci: In general we have also to deal with interrupted sulci [16] (see figure 2). In this case, we use the following procedure :

- If we note $p_{m}$ the maximum depth $\left(p_{m}=\max \left\{p_{0}, p_{1}, p_{2}\right\}\right)$, the sulci are resampled along the depth direction in order to obtain the same number of control points $\left(p_{m}\right)$ for all the sulci segments on their depth axis.

- Note $l_{m}=\max \left\{l_{1}+l_{2}, l_{0}\right\}$. If $l_{m}=l_{1}+l_{2}$, the sulcus 0 is resampled by a factor of $\frac{l_{m}}{l_{0}}$, and on the other hand sulci segments $i, i \in\{1,2\}$ are resampled by a factor of $\frac{l_{m}}{l_{1}+l_{2}}$.

On the figure 2, the curvilinear abscissa of point $M_{0}$ along the sulcus 0 length is $\frac{l_{1}}{l_{0}}$, after normalization between 0 and 1 . At $M_{0}$, the constraint field corresponding to the sulci matching is not contiguous.

When two homologous sulci are both interrupted, we match each segment as if they were continuous. This is possible because we have a labeling of each piece, Inferior-Superior (for the precentral sulcus for instance), or Antero-Posterior (for the superior temporal sulcus for instance). We have presented an approach for a sulcus described by two distinct segments, but this method can easily be extended if we have to deal with sulci having more segments.

Sparse Constraint Deformation Field: In every cases, we finally obtain two sulci each describes by $N$ control points. The sulcus of the source volume is described by a set of control points $S_{1}=\left\{C_{S}^{1} \ldots C_{S}^{N}\right\}$, when the homologous sulcus in the target volume is described by $S_{2}=\left\{C_{T}^{1} \ldots C_{T}^{N}\right\}$. For each point 


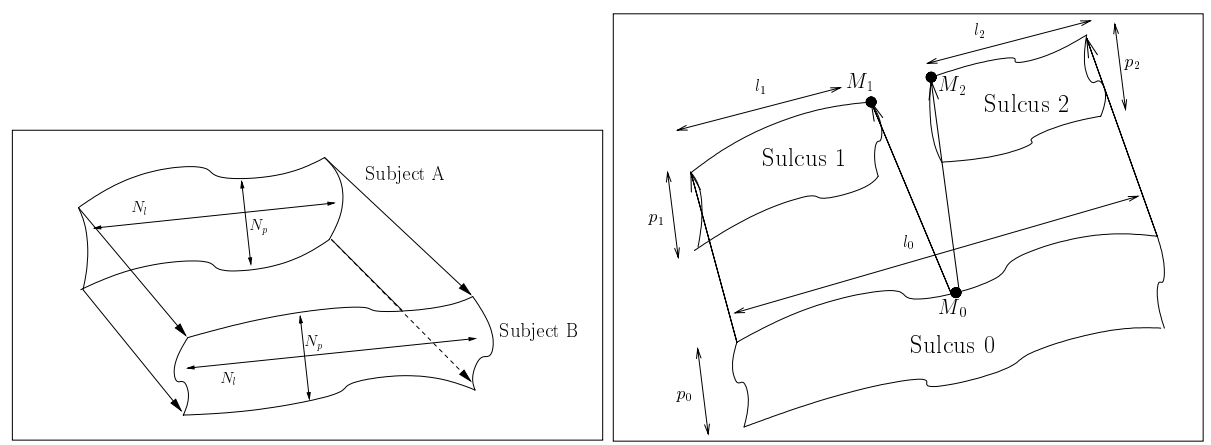

Fig. 2. Left: Matching of two homologous sulci. The sulcus with less control points is resampled in order to obtain the same number of control points on each axis, namely $N_{l}$ et $N_{p}$. Right: Matching of two sulci, with one of them interrupted. At $M_{0}$ (curvilinear abscissa $\frac{l_{1}}{l_{0}}$ ), the constraint field is discontinuous.

$S_{1}$, a constraint field can be explicitly computed $: \forall k \in\{1 \ldots N\}, \boldsymbol{w}_{k}^{c}=\overrightarrow{C_{S}^{k} C_{T}^{k}}$. Note $\mathcal{S}_{c}=S_{1}$ the kernel of the sparse constraint field.

Contrary to the matching approaches based on a distance measure (chamfer, ICP), this algorithm matches explicitly all sulci points. This mapping procedure may be discussed, because we do not know if it is anatomically plausible. Is it necessary to explicitly match the sulci extreme points? How to manage the sulci interruptions? In the absence of anatomical assumptions, it appeared more reasonable to us, in any cases in a primer stage, to implement this cortical mapping process. At the end of this process, we obtain a constraint field $\boldsymbol{w}^{c}$, which is defined on the support of source volume.

In order to reduce the sensitivity of the constraint field to sulci segmentation errors, we perform a non isotropic regularization of the field which can in a way be compared as a 3D adaptation of the Nagao filtering [15.

In the following, the approach we propose considers the existence of a field $\boldsymbol{w}^{c}$, and is therefore not limited to the incorporation of sulcal constraints.

\section{Integration of Sparse Constraints}

The sparse deformation field $\boldsymbol{w}^{c}$, deduced from the matching of cortical ribbons, must be integrated in the formulation of the registration problem. As in [6], we incorporate that information as a third energy term. The cost function therefore becomes:

$$
\begin{aligned}
& U\left(\boldsymbol{w} ; f, \boldsymbol{w}^{c}\right)=\sum_{s \in S}\left[\nabla f(s, t) \cdot \boldsymbol{w}_{s}+f_{t}(s, t)\right]^{2} \\
& +\alpha \sum_{<s, r>\in \mathcal{C}}\left\|\boldsymbol{w}_{s}-\boldsymbol{w}_{r}\right\|^{2}+\alpha^{c} \sum_{s \in \mathcal{S}_{c}}\left\|\boldsymbol{w}_{s}-\boldsymbol{w}_{s}^{c}\right\|^{2},
\end{aligned}
$$

where $\alpha^{c}$ is a parameter that balances the weight of the cortical constraint. 
The matching of cortical ribbons, as presented in the past section, might not be correct for all the points of the ribbons. As a matter of fact, there might be some segmentation errors, and these points should not be used as a hard constraint. Furthermore, we do not really know if it is anatomically correct to assume a one-to-one correspondence between the sulci. Therefore, we introduce a robust estimator $\psi_{3}$ on the cortical constraint term. The cost function is modified as:

$$
\begin{gathered}
\stackrel{\star}{U}\left(\boldsymbol{w}, \delta, \beta ; f, \boldsymbol{w}^{c}\right)=\sum_{s \in S} \delta_{s}\left(\boldsymbol{\nabla} f(s, t) \cdot \boldsymbol{w}_{s}+f_{t}(s, t)\right)^{2}+\psi_{1}\left(\delta_{s}\right) \\
+\alpha \sum_{<s, r>\in \mathcal{C}} \beta_{s r}\left(\left\|\boldsymbol{w}_{s}-\boldsymbol{w}_{r}\right\|\right)^{2}+\psi_{2}\left(\beta_{s r}\right) \\
+\alpha^{c} \sum_{s \in \mathcal{S}_{c}} \gamma_{s}\left(\left\|\boldsymbol{w}_{s}-\boldsymbol{w}_{s}^{c}\right\|\right)^{2}+\psi_{3}\left(\gamma_{s}\right) .
\end{gathered}
$$

Of course, the sparse constraint and the associated robust function introduce two new external parameters, $\alpha^{c}$ and $\psi_{3}$. However, these parameters are fixed with relative high values, as the constraints must be largely taken into account.

The minimization scheme is unchanged, compared to our previous work. We alternate between estimating the weights of the robust functions are estimating the deformation field. Once the weights are estimated and "frozen", the multigrid estimation of the field is performed through an iterative Gauss-Seidel scheme. Further details about that minimization strategy may be found in [7].

The local cortical constraint will have a relative spatial influence for two reasons. On the one hand, the standard regularization term propagates the local constraint because the minimization is alternated. One the other hand, the multigrid minimization, described in details in our previous work 7 makes it possible to estimate a deformation model on specified cubes, which will propagate the local constraint to a large group of voxels that compose the cube.

\section{Results on a Database of 18 Subjects}

To evaluate the benefits of cortical constraint, we have acquired a database of 18 subjects. For each subject, 6 main sulci per hemisphere have been extracted: central, precentral, postcentral, sylvian, superior temporal and superior frontal. Among the 18 subjects, one has been chosen as a reference subject, and all the subjects have been registered onto that subject, with the previous method and the constrained one. For both methods, we keep the same set of parameters for all the subjects, showing that the algorithm is quite robust with respect to parameter settings.

We have designed global and local measures to assess the quality of the registration, and to compare the original method to the constrained one. 


\subsection{Global Measures}

Once every subject of the database has been mapped into the reference subject, it is possible to compute an average volume, by normalizing the 17 deformed volumes. Note that all the volumes are computed with the estimated deformation field and trilinear interpolation. The results are presented on figure 3 with axial, sagittal and coronal views for both methods.

Looking at cortical areas, we can see that the average volume is less blurred and more similar to the reference volume. On other regions, the results are identical.

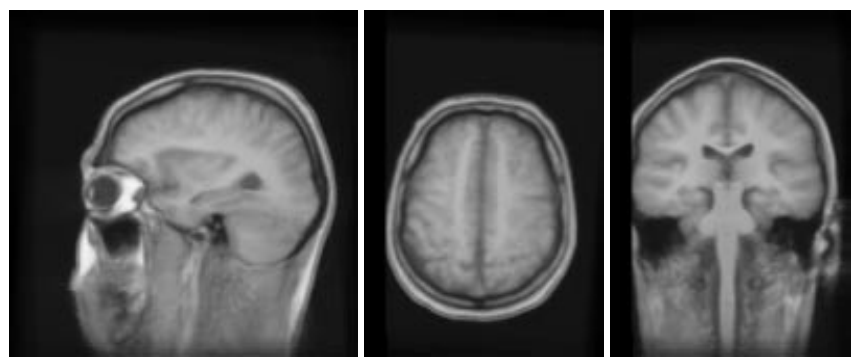

Previous work

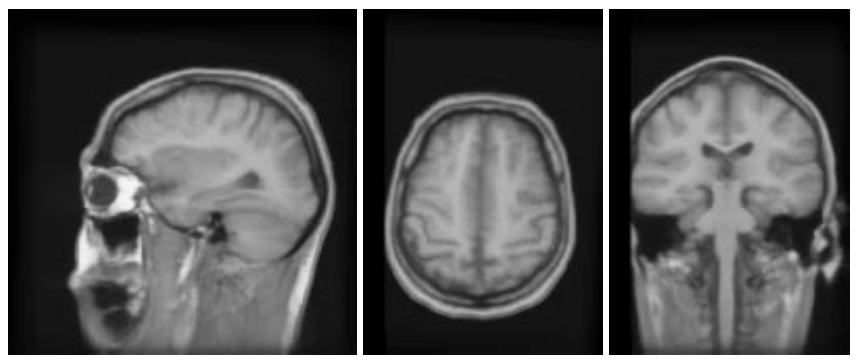

Constrained registration

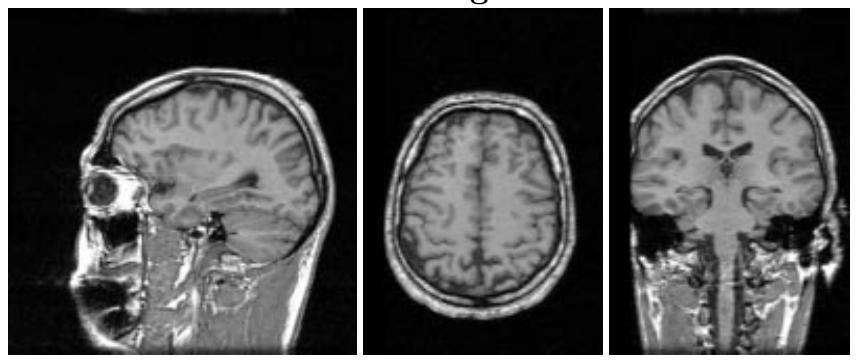

Reference subject

Fig. 3. Average volumes, computed with the 17 deformed subjects. The volume corresponding to the constrained method is more precise on the cortex. 
In order to assess numerically the difference between the methods, we compute the mean square error (MSE 1 ) between the averaged volumes and the reference volume. We compute two average errors: one for all the voxels of the volume, and one restricted to the segmentation mask of the reference brain. Results are presented on table 1, and show the benefit of the cortical constraint, as the error decreases by $25 \%$. The MSE might not be a good measure when considering only two subjects. Notwithstanding that, it is valuable when comparing the average volume (computed with 17 registered volumes) to the reference volume, and is also valuable when comparing two different methods.

Table 1. Mean Square error (MSE) between the average volume and the reference volume. We present two mean errors: the first one is computed for all the voxels of the volume, whereas the second one is computed only on the segmentation mask of the reference brain. The benefit of the cortical constraint is visible, as we can see a $25 \%$ decrease of the error.

\begin{tabular}{|c|c|c|}
\hline & $\begin{array}{c}\text { Computation for } \\
\text { all the voxels }\end{array}$ & $\begin{array}{c}\text { Computation restricted to } \\
\text { the segmentation mask of the brain }\end{array}$ \\
\hline Previous work & 750.4 & 624.5 \\
\hline Constrained registration & 545.2 & 506.7 \\
\hline
\end{tabular}

At that stage, the evaluation is not fair, as the criterion is more or less related to the similarity measure that is used to drive the registration process. Therefore, we design another "global" evaluation of the registration based on the registration of anatomical features. For each subject, we have the classification of the cortex into grey matter and white matter, obtained with the method [9]. We compare the classification of each subject, projected into the reference subject, and the reference classification, by computing overlapping measure such as sensitivity, specificity, and total performance. For concision sake, we keep the total performance measure, and compute the mean and the deviation of that measure over the database of subjects. Results are presented on table 2 , The results are comparable for the two techniques, but the deviation of the constrained technique is lower, meaning that the algorithm is more robust.

\subsection{Local Measures}

In that section, we want to measure locally the benefits of the cortical constraints. Therefore, we designed measures to evaluate the quality of the matching of sulci. Looking at the figure 1 one can see the sulci of the reference subject, $\mathrm{R}$, and two deformed sulci 1 and 2. The two sulci should ideally match the reference sulci, and which one is the best deformed sulci? If we consider the global positioning,

${ }^{1} M S E=\frac{1}{N} \sum_{i=1}^{i=N}\left(I_{1}(i)-I_{2}(i)\right)^{2}$, where $I_{1}$ and $I_{2}$ are the volumes to compare, and $N$ is the number of voxels. 
Table 2. Overlapping measures between grey/white matter. For each subject, the classification of the cortex is projected into the reference subject and compared to the classification of the reference subject. We choose an overlapping measure defined by the total performance, and compute the mean and deviation of that measure over the database of subjects.

\begin{tabular}{|c|c|c|c|}
\hline & Tissue & Mean & Deviation \\
\hline Previous work & grey & 93.5 & 0.07 \\
& white & 95.1 & 0.07 \\
\hline Constrained registration & grey & 93.5 & 0.05 \\
& white & 95.3 & 0.05 \\
\hline
\end{tabular}

sulcus 1 seems to be the best, but if we consider the shape, sulcus 2 appears to be better deformed. In that section, we first visualize the deformed sulci, then we will evaluate numerically the registration of sulci, with respect to global positioning an with respect to shape recovering.

Visualization of Deformed Sulci: If we consider one particular sulcus over the database of 18 subjects, one can deform each sulcus with the estimated deformation field and look at the superposition with the reference sulcus. The visualizations, for the central left sulcuss and the left sylvian fissure are presented on figures 4 and 5 respectively. One can observe the variability of the registered sulci with the previous method, and the way sulci are registered with the modified method.

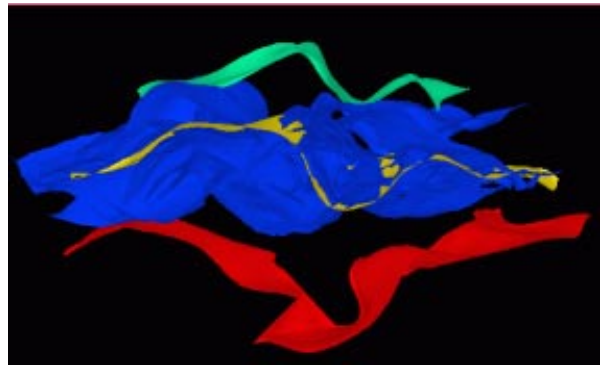

Previous method

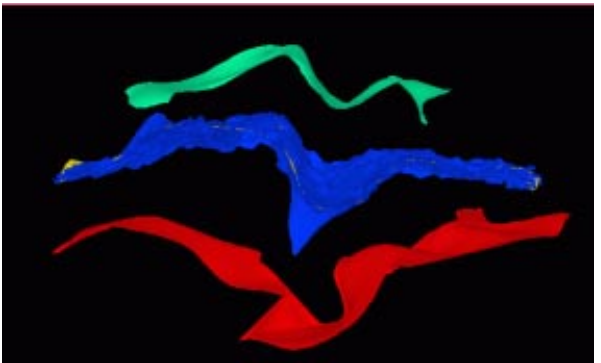

Constrained registration

Fig. 4. The left central sulci of each subject (in blue) are deformed toward the reference subject, and compared to the left central sulcus of the reference subject (in yellow). For the reference subject, we have also traced the left precentral sulcus in red and the left postcentral sulcus in green. The constrained method leads to a significantly lower variability, compared to the previous method. 


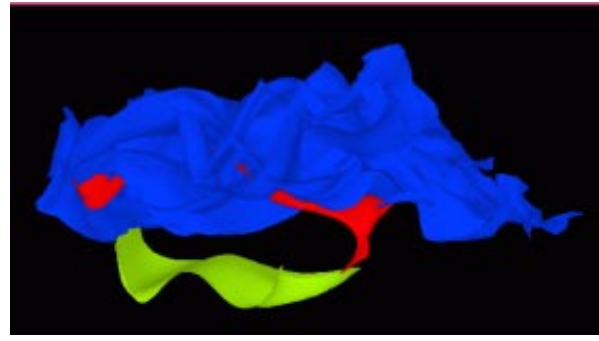

Previous method

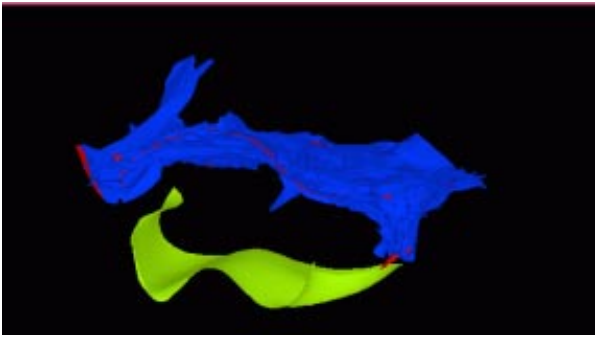

Constrained registration

Fig. 5. The left sylvian sulci of each subject (in blue) are deformed toward the reference subject, and compared to the left sylvian sulcus of the reference subject (in red). We have also traced the left superior temporal sulcus of the reference subject in green.

Average Sulcus As the sulci are defined by their control points, it is possible to compute, after resampling, an average sulcus with the deformed sulci of each subject. The control points of the average sulcus are the mean of the corresponding control points of the deformed sulci. We can then visualize the average sulcus and compare it to the reference sulcus. The figure 6 present the average sulci for the central left sulcus and the left sylvian fissure. We observe that the average sulci obtained with the constrained method are almost stuck to the reference sulci, thus showing that the local constraint has been well integrated in the registration process.

\section{Numerical Evaluation}

Euclidean distances between sulci: In that section, we intend to assess numerically the impact of the registration on the matching of sulci. As the sulci are defined by their control points, we can first compute a mean distance between sulci after resampling. However, the distance does not reflect how shapes are similar, therefore we also perform a principal component analysis (PCA) to evaluate the similarity of shape.

For each subject, and for each sulcus, we compute a distance between the deformed sulci of one subject and the corresponding sulci of the reference subject. We then average the distances, for the 18 subjects and for the 12 sulci (per subject), and present the results in table 7 . The constrained method gives significantly better results, with an average distance that is equivalent to the resolution of MR images. We also evaluate the global positioning of sulci, by computing a distance between the center of gravity of the sulci. For the constrained method, the alignment is good as the mean distance between the registered sulci is 0.2 voxels. 

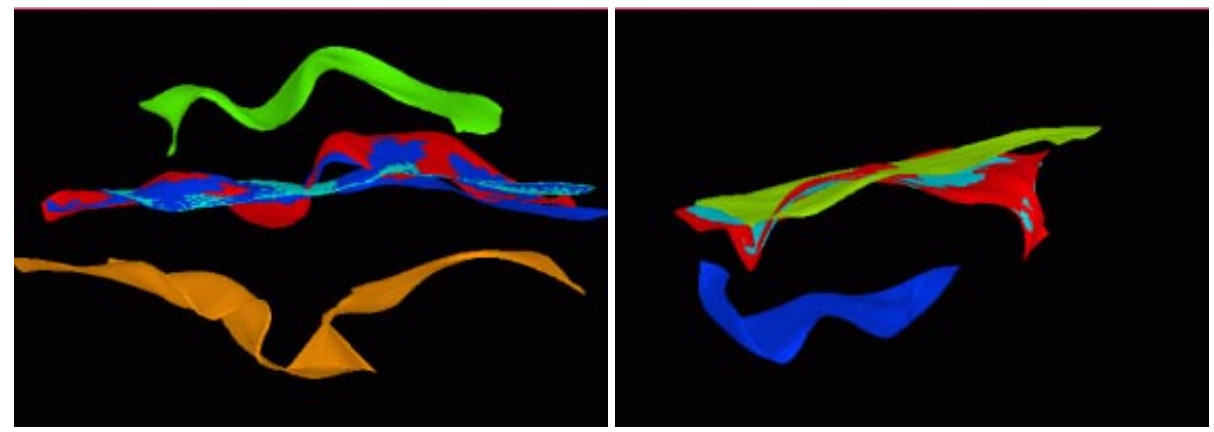

Fig. 6. Left: For the left central sulcus, the average sulcus obtained with the previous method is drawn in cyan, while the average sulcus obtained with the constrained method is drawn in blue. We have also put three sulci of the reference subject, the left central in red, the left postcentral in green and the left precentral in orange. Right: For the left sylvian sulcus, the average sulcus obtained with the previous method is drawn in green, while the average sulcus obtained with the constrained method is drawn in blue. We have also put two sulci of the reference subject, the left sylvian in red, the left temporal superior in blue.

Principal component analysis: However, the distance between sulci does not reflect how shapes are similar. The principal component analysis makes it possible to define a metric that is adapted to the comparison of shapes. We will not describe here the PCA technique, thus referring the reader to [5] for further information.

For each method, the population of shapes is composed by a sulcus of the reference subject, and the corresponding sulci of each subjects, deformed toward the reference subject by a given registration method. We consider the trace of the covariance matrix, because it reflects the total amount of variation along the axis of the decomposition, and because the trace is invariant. The table $\mathbf{7}$ compares the traces obtained with the previous method and the constrained registration, for three different sulci: the left central, the left frontal superior and the left sylvian. The constrained method gives significant better results, as the trace is almost divided by a factor 10 .

\section{Conclusion}

We have presented in that paper a cooperation method between an "iconic" registration method, and a landmark based registration. We have chosen to incorporate sparse sulcal constraints, because sulci are relevant landmarks, from a functional and morphological point of view. The sulci are extracted with the active ribbon method, presented in [10]. The energy-based framework [7] makes 


\begin{tabular}{|c|c|c|c|c|c|}
\hline & Sulcus & Method & Trace \\
\hline & & & Central & $\begin{array}{c}\text { Previous method } \\
\text { Constrained registration }\end{array}$ & $\begin{array}{c}11136 \\
1472\end{array}$ \\
\hline & $\begin{array}{c}\text { Mean distance } \\
\text { between } \\
\text { control points }\end{array}$ & $\begin{array}{c}\text { Mean distance } \\
\text { between } \\
\text { center of gravity }\end{array}$ & Superior frontal & \begin{tabular}{|c|} 
Previous method \\
Constrained registration
\end{tabular} & $\begin{array}{c}11595 \\
2273 \\
\end{array}$ \\
\hline Previous method & 10.4 & 8.0 & \multirow[t]{2}{*}{ Sylvian } & Previous method & 17760 \\
\hline Constrained registration & 1.2 & 0.2 & & Constrained registration & 2022 \\
\hline
\end{tabular}

Fig. 7. Left: Average distances between registered sulci. The mean is performed for all the sulci (12 per subject) and for all the subjects (18). We compute two distances, one between the control points and the other between the center of gravity. Right: For three populations of deformed sulci of the left hemisphere, the trace of the covariance matrix reflects the total amount of variation that is observed along the axis of the decomposition. The trace is invariant, which makes it possible to compare the results of both methods.

it possible to incorporate naturally the local sparse constraints and to express the complete registration problem in the same formalism.

On a database of 18 subjects, on which 6 major sulci have been extracted by hemisphere for each subject, we have demonstrated the significant impact of sparse constraints. We have designed global measures (average volume, mean error, overlapping of brain tissues), as well as local measures (distance between sulci after registration, shape comparison based on PCA). Both measures have shown the impact of cortical constraints.

\section{Acknowledgements}

This work has been partly supported by the Brittany Country Council under a contribution to the student grant. Grant support for the acquisition of the data was provided by the GIS Project "cognition science".

\section{References}

1. A. Caunce and CJ. Taylor. Using local geometry to build 3D sulcal models. Proc. IPMI, pages 196-209, 1999.

2. H. Chui, J. Rambo, J. Duncan, R. Schultz, and A. Rangarajan. Registration of cortical anatomical structures via robust 3D point matching. Proc. IPMI, pages 168-181, 1999.

3. L. Collins, G. Le Goualher, and A. Evans. Non linear cerebral registration with sulcal constraints. Proc. of MICCAI, pages 974-985. Springer, October 1998.

4. L. Collins, G. Le Goualher, R. Venugopal, A. Caramanos, A. Evans, and C. Barillot. Cortical constraints for non-linear cortical registration. Proc. VBC, pages 307-316, September 1996.

5. T. Cootes, C. Taylor, D. Hooper, and J. Graham. Active shape models- their training and application. CVIU, 61(1):31-59, 1995. 
6. J. Gee, L. Le Briquer, C. Barillot, and D. Haynor. Probabilistic matching of brain images. In Bizais et al., editor, Proc. Information Processing in Medical Imaging, Brest, June 1995. Kluwer academic publisher.

7. P. Hellier, C. Barillot, E. Mmin, and P. Prez. An energy-based framework for dense $3 \mathrm{~d}$ registration of volumetric brain image. In IEEE CVPR, pages 270-275, Jun 2000.

8. B. Horn and B. Schunck. Determining optical flow. Artificial Intelligence, 17:185203, 1981.

9. F. Lachmann and C. Barillot. Brain tissue classification from mri data by means of texture analysis. In SPIE Press, editor, Spie Medical Imaging, pages 72-83, 1992.

10. G. Le Goualher, C. Barillot, and Y. Bizais. Modeling cortical sulci with active ribbons. IJPRAI, 8(11):1295-1315, 1997.

11. G. Le Goualher, E. Procyk, L. Collins, R. Venegopal, C. Barillot, and A. EvansG. Automated extraction and variability analysis of sulcal neuroanatomy. In IEEE TMI, 18(3):206-217, Mars 1999.

12. J. Maintz, MA. Viergever. - A survey of medical image registration. Medical Image Analysis, 2(1):1-36, 1998.

13. J.-F. Mangin, V. Frouin, I. Bloch, J. Regis, and J. Lpez-Krahe. From 3d magnetic resonance images to structural representations of the cortex topography using topology preserving deformations. Journal of Math. Ima. and Vis., 5(4):297-318, 1995.

14. J. Mazziotta, A. Toga, A. Evans, P. Fox, and J. Lancaster. A probabilistic atlas of the human brain: theory and rationale for its development. Neuroimage, 2:89-101, 1995.

15. M. Nagao and M. Matsuyama. Edge preserving smoothing. CVGIP, 9:394-407, 1979.

16. M. Ono, S. Kubik, C. Abernathey. - Atlas of the cerebral sulci. - Verlag, 1990.

17. D. Riviere, JF. Mangin, D. Papadopoulos-Orfanos, J. Martinez, V. Frouin, and J. Regis. Automatic recognition of cerebral sulci using a congregation of neural networks. In Proc. of MICCAI, 2000.

18. N. Royackkers, H. Fawal, M. Desvignes, M. Revenu, and JM. Travere. Morphometry and identification of brain sulci on three-dimensional MR images. Proc. IPMI, pages 379-380, June 1995.

19. S. Sandor and R. Leahy. Surface-based labeling of cortical anatomy using a deformable atlas. IEEE TMI, 16(1):41-54, 1997.

20. G. Szekely, Ch. Brechbhler, O. Kbler, R. Ogniewicz, and T. Budinger. Mapping the human cerebral cortex using $3 \mathrm{~d}$ medial manifolds. In $V B C$, pages $130-144$, 1992. SPIE.

21. J. Talairach and P. Tournoux. Referentially oriented cerebral MRI anatomy. Georg Thieme Verlag, New-York, 1993.

22. J.P. Thirion and A. Gourdon. The marching lines algorithm: new result and proofs. Research report 1881, INRIA, March 1993.

23. P. Thompson and A. Toga. A surface-based technique for warping threedimensional images of the brain. IEEE TMI, 15(4):402-417, 1996.

24. M. Vaillant and C. Davatzikos. Hierarchical matching of cortical features for deformable brain image registration. Proc. IPMI, pages 182-195, June 1999.

25. X. Zeng, L.H. Staib, R.T. Schultz, H. Tagare, L. Win, and J.S. Duncan. A new approach to 3D sulcal ribbon finding from MR images. MICCAI, pages 148-157, September 1999. 